\title{
Análise dos fatores que levam mulheres médicas a não optarem por especialidades cirúrgicas
}

\author{
Analysis of the factors that lead medical women to not opt for surgical specialties
}

\author{
Daiane Paulo', Mariana da Silva Assis², Maria Regina Orofino Kreuger ${ }^{3}$
}

Paulo D, Assis MS, Kreuger MRO. Análise dos fatores que levam mulheres médicas a não optarem por especialidades cirúrgicas / Analysis of the factors that lead women physicians not to choose surgical specialties. Rev Med (São Paulo). 2020 2020 maio-jun.;99(3):230-5.

\begin{abstract}
RESUMO: Objetivo: Verificar as prováveis causas que levam as mulheres médicas a não optarem por especialidades cirúrgicas. Métodos: foram avaliadas as respostas de 75 médicas, através de um questionário objetivo composto de 15 perguntas sobre a não escolha da área cirúrgica,que foram transformadas em porcentagem e apresentadas em gráficos e tabelas. Resultados: Observou-se que $45,3 \%$ das médicas já cogitaram ser cirurgiãs, que o fator mais assinalado como limitante, por $40 \%$ das médicas (questão de múltipla escolha), à escolha da área cirúrgica foi o horário de plantões/sobreaviso. Além disso, observou-se, nesta pesquisa, que $86,7 \%$ acreditam que é possível ser cirurgiã e constituir família. A característica de personalidade mais utilizada por $72 \%$ das médicas participantes deste estudo para definir uma mulher cirurgiã foi: "forte". E apenas $12 \%$ das participantes não acreditam que haja machismo/preconceito por parte dos colegas ou pacientes em relação às cirurgiãs. Conclusão: Além de identificar os possíveis fatores que limitam as mulheres médicas a optarem por especialidades cirúrgicas, conclui-se que mais estudos como este devem ser feitos para que questões laborais (flexibidade de horários, igualdade salarial) e comportamentais (preconceito, machismo) sejam revistas.
\end{abstract}

Descritores: Médicas/estatísticas \& dados numéricos; Medicina; Mulheres/educação.

\begin{abstract}
Objective: To verify the probable reasons that lead medical women to not opt for surgical specialties. Methods: We evaluated the answers of 75 medical women, through an objective questionnaire composed of 15 questions about their choice to not go into surgical specialties. The data was then transformed into percentages and shown in charts and tables. Results: We observed that $45.3 \%$ of the medical women have already considered being a surgeon. The most limiting factor; $40 \%$ of the participants chose was on call hours. Besides that, we observed that $86.7 \%$ believe that it is possible to be a surgeon and institute a family. The characteristic most used to define a female surgeon was "strong", by $72 \%$. And only $12 \%$ of participants believe that there isn't prejudgement by colleagues or patients about women surgeons. Conclusion: In addition to identifying the probable factors that limit women to opt for surgical specialties, we concluded that more studies like this one should be done, in order to review laboral issues (flexibility of turns, equal pays) and behaviors (prejudice, sexism).
\end{abstract}

Keywords: Physicians, women/statistics \& numerical data; Medicine; Women/education.

1. Universidade do Vale do Itajaí. Acadêmica do Curso de Medicina, Itajaí, SC, Brasil. ORCID: https://orcid.org/0000-0001-5694-2505.Email: daaai_@ hotmail.com.

2. Universidade do Vale do Itajaí. Acadêmica do Curso de Medicina, Itajaí, SC, Brasil. . ORCID: https://orcid.org/0000-0001-7891-2083. Email: maryassys@hotmail.com.

3. Universidade do Vale do Itajaí. Doutora em Patologia pela Universidade de São Paulo (USP). ORCID: https://orcid.org/0000-0003-0811- 948X. Email: orofinokreuger@gmail.com.

Endereço para correspondência: Daiane Paulo. Rua Alcides Nascimento, 19. São Vicente, Itajaí, SC. CEP:88309-124. Email: daaai_@hotmail.com 


\section{INTRODUÇÃO}

$\mathrm{N}^{\mathrm{o}}$ o decorrer da história, a Medicina não se conteve apenas em enfatizar que homens e mulheres são diferentes, mas em qualificar essa diferença, geralmente, tentando enfatizar inaptidões femininas ${ }^{1}$. Algumas profissões sempre foram consideradas atividades exclusivas de homens, entre elas a medicina ${ }^{2}$. O preconceito sobre as atividades laborais das mulheres em determinadas áreas existe há muitos séculos. Na área médica, a mulher foi proibida de estudar em uma escola de Medicina até 1879, de modo que a primeira médica do Brasil se formou na Faculdade de Medicina da Bahia, em $1887^{3}$.

Até o início do século XIX, quando o exercício da Medicina ainda não dependia de licenças dadas por cursos oficiais, que não aceitavam mulheres, há várias referências a mulheres cirurgiãs que trabalhavam no anonimato ou escudadas por parentes masculinos ${ }^{4}$. $\mathrm{O}$ caso mais notório e curioso é o de Miranda Barry (1797-1865), que, sob o nome de James Barry, foi o principal Oficial Médico e Cirurgião da Armada Britânica, onde serviu durante 40 anos $^{4}$.

Entre 1949 e 1968, as mulheres representavam, em média, $10 \%$ de cada turma; entre 1982 e 1993, esse percentual já chegava aos $50 \%{ }^{4}$. Entretanto, o aumento percentual de cirurgiãs não acompanhou o crescimento acelerado do número de médicas formadas nas três últimas décadas ${ }^{4}$. Explicar o porquê de poucas mulheres desejarem ser cirurgiãs é um tema complexo que não tem resposta única ${ }^{5}$. Verifica-se, assim, a tendência consistente de feminização da medicina no Brasil, que se observa ao longo das últimas décadas e que se acentuou recentemente ${ }^{6}$. No período de 2000 a 2016, foram registrados 220.993 novos médicos, dos quais 49,3\% eram homens; $50,7 \%$, mulheres. Quando se observa essa distribuição por ano de entrada, vê-se que a porcentagem de mulheres médicas cresce rapidamente ${ }^{7}$.

Uma certa falta de auto-confiança feminina e de modelos estimulantes de cirurgiãs de sucesso, além dos problemas advindos da falta usual de suporte institucional às mães médicas, são as dificuldades a serem vencidas pelas mulheres que desejam ser cirurgiãs, fatores ainda presentes, contribuintes para o pequeno número de mulheres cirurgiãs ${ }^{4}$.

Durante séculos, a mulher lutou, com muita fibra, para alcançar o direito natural de exercer a profissão médica, contra todas as hostilidades machistas de sempre ${ }^{8}$. Mesmo diante de tantas barreiras, já existem alguns estudos que trazem dados favoráveis às cirurgiãs quando comparadas aos cirurgiões masculinos. Em um estudo realizado na Universidade de Toronto (Canadá), verificou-se que pacientes de médicas têm $12 \%$ menos chance de morrer em um período de até 30 dias pós-procedimento 9 . Outro estudo publicado na revista da Associação Catarinense de Medicina ${ }^{10}$, sobre a prevalência de erros médicos nos processos julgados pelo Tribunal Regional de Medicina de Santa Catarina, mostra que os médicos condenados por erro médico, em sua maioria, são do gênero masculino e trabalhavam em serviço cirúrgico.

Qualquer esforço para corrigir a má distribuição de profissionais por especialidade deve incluir as mulheres, procurando descobrir quais são os fatores que influenciam as preferências na escolha da especialidade por parte das estudantes de Medicina e entender por que as mulheres não têm sido atraídas para determinadas especialidades ${ }^{5}$.

\section{MÉTODO}

Estudo prospectivo e qualitativo, no qual as participantes foram convidadas a responder um questionário com 15 questões de múltipla escolha com o intuito de analisar quais os fatores que levavam as mulheres médicas a não optarem pelas especialidades cirúrgicas. Para atingir este objetivo, utilizou-se como instrumento o questionário intitulado: “Análise dos fatores que levam as mulheres médicas a não optarem pelas especialidades cirúrgicas", o qual foi adaptado da pesquisa intitulada: "Fatores influenciadores da escolha da especialidade por médicos residentes de Minas Gerais", de Patrícia Roberta Berithe Pedrosa de Oliveira (UNICAMP).

A amostra inicial foi de 100 médicas, de modo que, ao final, foram avaliados 75 questionários respondidos por médicas não cirurgiãs atuantes na cidade de Itajaí-SC e que pertencem a uma ou mais das seguintes especialidades: Clínica Médica, Pediatria, Medicina de família, Medicina preventiva, Endocrinologia e Metabologia, Genética Médica, Hematologia, Homeopatia, Infectologia e Patologia ou que sejam médicas generalistas.

Haja vista o exposto acima e segundo os objetivos deste estudo, foram usados como critérios de inclusão: médicas com registro na cidade de Itajaí-SC e que pertencessem às especialidades supracitadas, as quais, segundo dados do Conselho Federal de Medicina, são especialidades compostas predominantemente por mulheres. Os critérios de exclusão foram o preenchimento incompleto do questionário e/ou a participante não pertencer a nenhuma das especialidades supracitadas nos critérios de inclusão. Dos 25 questionários excluídos deste estudo, em 23 deles, as médicas participantes deixaram uma ou mais questões em branco, e em dois deles, as médicas não se enquadravam nas especialidades supracitadas nos critérios de inclusão.

Os dados obtidos pelo questionário foram anotados e agrupados em uma planilha do Microsoft Office Excel, transformados em porcentagens com intervalo de confiança de $95 \%$ (o que caracteriza uma probabilidade de erro de 0,05 ). Os resultados obtidos foram apresentados na forma de tabelas e/ou gráficos.

A amostra foi do tipo não probabilística, constituída 
por conveniência, isto é, por sujeitos de pesquisa que, de livre e espontânea vontade, aceitaram participar deste estudo. Não foram feitas quaisquer distinções quanto aos hábitos sociais, e as respostas foram anônimas. O Comitê de Ética e Pesquisa da UNIVALI aprovou o estudo e todas as participantes preencheram formulários de Consentimento Livre e Esclarecido. (CAAE: 78984817.9.0000.0120)

Não houve nenhum conflito de interesse e nenhuma fonte financiadora.

\section{RESULTADOS}

A amostra do estudo foi constituída por 75 (setenta e cinco) médicas pertencentes às seguintes especialidades: Clínica Médica, Medicina da Família e da Comunidade, Endocrinologia, Pediatria, Hematologia, Infectologia e, ainda, médicas Generalistas. Da amostra, 45,33\% graduaram-se entre os anos 2010 e $2017 ; 29,33 \%$, entre 2000 e $2009 ; 12 \%$, na década de $90 ; 12 \%$, na década de 80 e $1,33 \%$, nos anos 70 .

Quando questionadas sobre em que momento da graduação decidiram qual especialidade queriam seguir, $46,66 \%$ das médicas responderam que foi no final do curso, enquanto $88 \%$ das médicas afirmaram que durante a graduação já pensaram em escolher outra especialidade, e cerca de metade das participantes apontaram que já cogitaram seguir a área cirúrgica. A Figura 1 ilustra o número de médicas que pensaram em seguir a área cirúrgica durante a graduação.

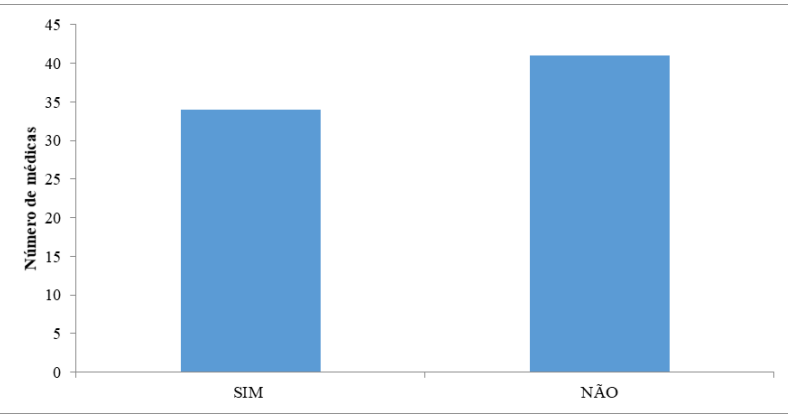

Figura 1. Número de médicas que já cogitaram seguir a área cirúrgica durante a graduação

Entretanto, 92\% das voluntárias da pesquisa afirmaram que, atualmente, sentem-se satisfeitas com a especialidade escolhida.

Quando questionadas sobre o porquê de não optarem por seguir a área cirúrgica, as médicas, em uma questão de múltipla escolha, puderam marcar diversos fatores, dentre os quais a demanda serviçofamília e os horários de plantões e sobreavisos foram os mais assinalados. A Tabela 1 ilustra os possíveis fatores assinalados pelas médica como sendo limitantes à escolha da área cirúgica.
Tabela 1 - Fatores que levaram as médicas a não optarem pelas especialidades cirúrgicas

\begin{tabular}{|lc|}
\hline Fatores & $\mathbf{N}^{0}$ de médicas \\
\hline Horário de plantões & 30 \\
\hline Demanda serviço família & 29 \\
\hline Sempre quis ser clínica & 26 \\
\hline Pressão do dia-a-dia & 23 \\
\hline Outros & 22 \\
\hline Estresse e cansaço & 15 \\
\hline Preconceito com mulheres da área & 12 \\
\hline Baixo desempenho na área & 7 \\
\hline Conselho de professores & 2 \\
\hline
\end{tabular}

Nota: * As médicas puderam escolher entre mais de um fator pelo qual ela decidiu não seguir a especialidade cirúrgica (Questão múltipla escolha).

Além disso, 49,3\% das médicas acreditam que para que uma cirurgiã seja bem sucedida profissionalmente, ela deve abdicar de muitas escolhas pessoais (Figura 2).

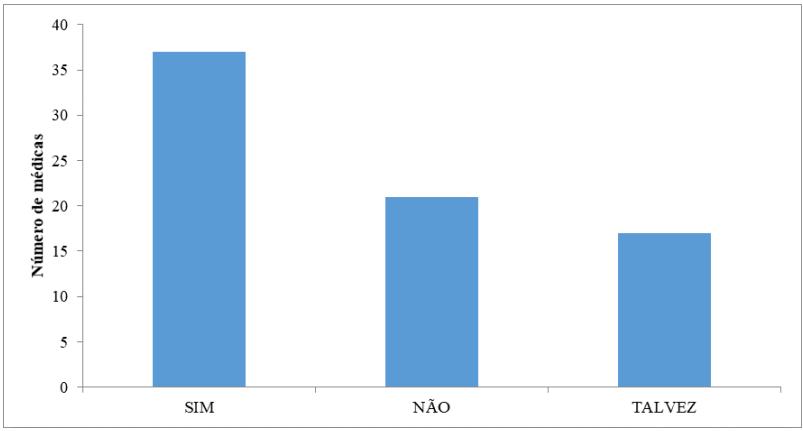

Figura 2-Respostas das voluntárias a respeito do questionamento se é necessário que as cirurgiãs abdiquem de muitas escolhas pessoais para serem bem sucedidas profissionalmente

Observou-se, através da resposta das médicas ao questionário, que grande parte das participantes da pesquisa acredita que existe a possibilidade de ser cirurgiã e constituir família simultaneamente (Figura 3).

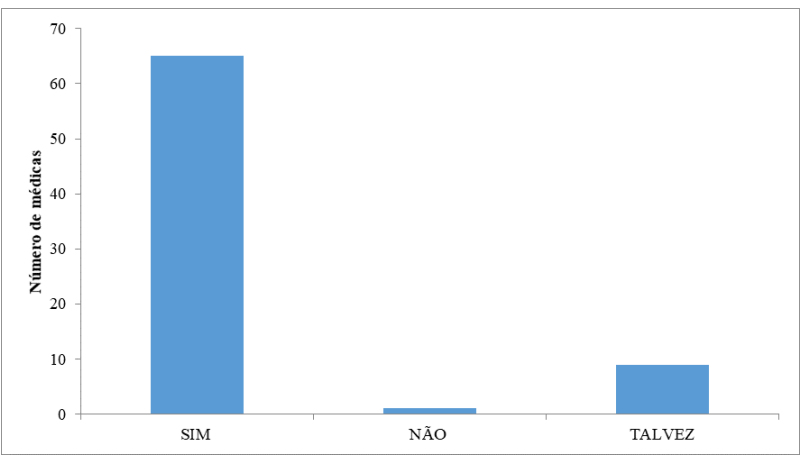

Figura 3 - Respostas das voluntárias a respeito do questionamento se é possível ser cirurgiã e constituir família 
As médicas participantes, quando questionadas a respeito da personalidade das cirurgiãs, consideraram, na sua maioria, que estas são fortes e corajosas (Figura 4).

Verificou-se, ainda, que a falta de cirurgiãs mentoras que sejam modelos de referência a serem seguidos durante a graduação não é um fator que influencie a não escolha por uma área cirúrgica, segundo as participantes do estudo em questão.

Dentre todas as participantes da pesquisa, apenas $12 \%$ acreditam que não há machismo por parte dos pacientes ou dos colegas de trabalho com as médicas cirurgiãs (Figura 5).

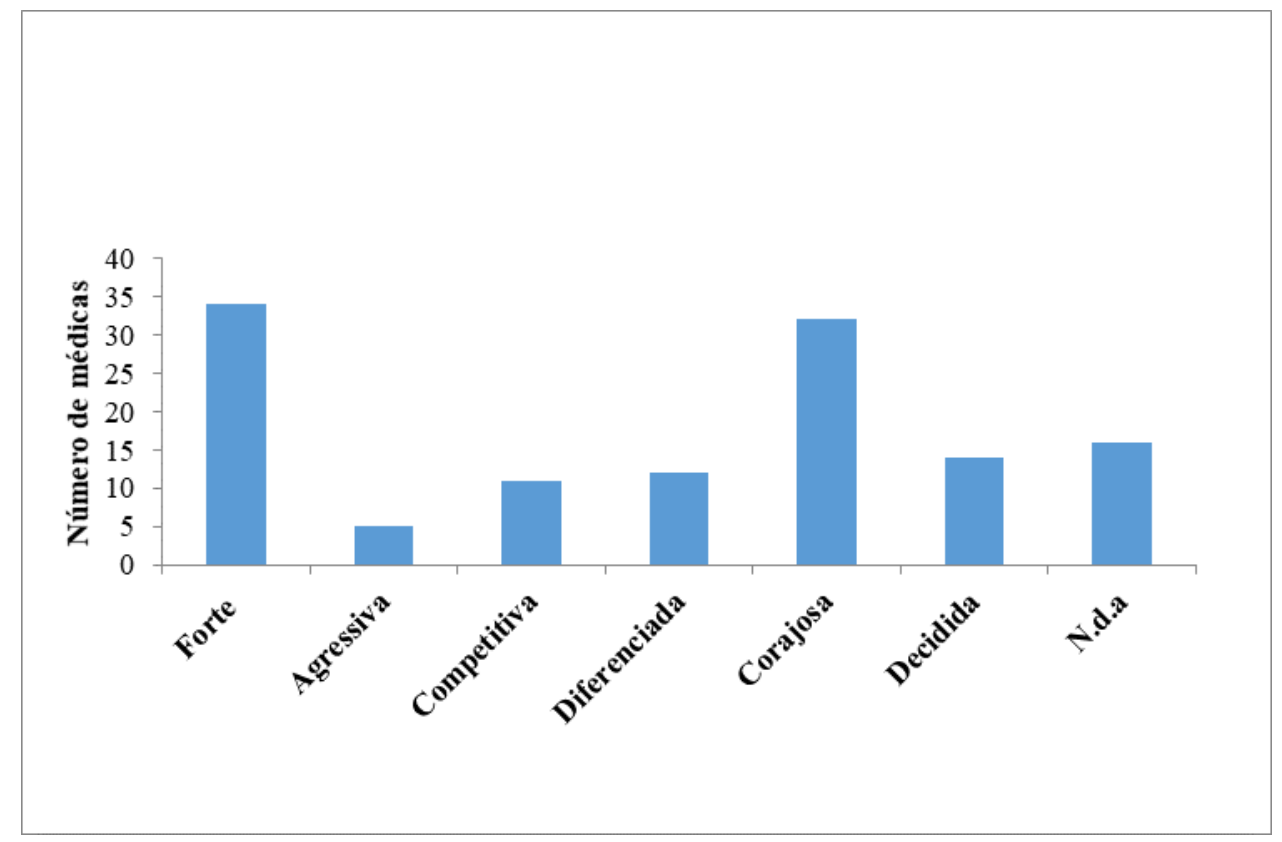

Nota: * A questão era de múltipla escolha, logo uma médica poderia marcar mais de uma característica

Figura 4 - Características marcadas pelas participantes do estudo para qualificar a personalidade de uma médica cirurgiã

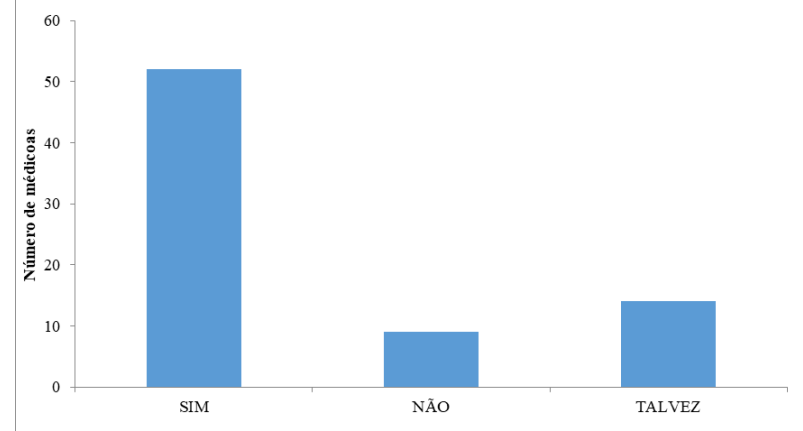

Figura 5 - Respostas das voluntárias a respeito do questionamento se há machismo, preconceito ou discriminação por parte de pacientes ou colegas de trabalho em relação a mulheres cirurgiãs

\section{DISCUSSÃO}

Explicar por que poucas mulheres desejam ser cirurgiãs é um tema complexo para o qual não tem resposta única. Em pleno século XXI, mulheres ainda são questionadas sobre a vontade de ter filhos e planos pessoais, como se tal fato fosse interferir negativamente na sua formação, enquanto os homens, quando concorrentes às mesmas vagas, não são questionados de tais intenções ${ }^{12}$. Diferentemente dos homens, que, simplesmente, escolhem uma profissão ou especialidade que lhes agrade ou convenha, as mulheres, mesmo quando mais capazes, têm inúmeros obstáculos a vencer ${ }^{4}$. Nesta pesquisa, em uma pergunta de múltipla escolha sobre fatores que levam à não opção pela área cirúrgica, com nove opções de respostas, os resultados mais expressivos, que ficaram em primeiro e segundo lugar, respectivamente, foram o horário dos plantões/sobreaviso e demanda serviço-família, o que corrobora a literatura supracitada, demonstrando que há muitos empecilhos a serem vencidos pelas mulheres que desejam ser cirurgiãs.

Mesmo em Cirurgia Geral, que é uma das especialidades básicas, as mulheres ocupam apenas um quinto do total ${ }^{11}$. Neste estudo, $45,3 \%$ das participantes afirmaram, ao responder o questionário, que jamais cogitaram ser cirurgiãs. Cuidados com a casa e com os filhos são ainda tidos como papéis femininos. Um homem médico se dedicando com exaustão a sua especialidade é visto como um indivíduo que quer o melhor para si e seus pacientes. Já uma mulher médica é vista como alguém que está abandonando a casa, deixando os filhos 
para segundo plano ${ }^{13}$. Confundem com frequência os conceitos de materno e feminino, capacidade de cuidado e abstração. Por consequência, direcionam as mulheres para atividades que pareceriam naturais e desejadas, propondo uma conciliação entre a condição feminina e o exercício profissional $^{12}$.

Cruzando-se os dados obtidos nas respostas das perguntas, em uma das questões abordadas, a maioria das médicas respondeu que um dos fatores preponderantes para a não escolha da área cirúrgica foi a dificuldade de conciliar a demanda serviço-família. Posteriormente, quando questionadas se acreditavam que existiria a possibilidade de serem cirurgiãs e constituírem família, as participantes da pesquisa, em sua maioria, responderam que sim. Um dos possíveis fatores aventados como influenciador à não escolha das mulheres pela área cirúrgica, de senso comum e crítico, é o desafio de administrar a carreira e família. O Jornal Americano de Cirurgia traz como fatores hipotéticos para explicar a pouca quantidade de mulheres cirurgiãs em cargos de chefia: as responsabilidades familiares que a mulher tem, a falta de mentoras cirurgiãs ${ }^{14}$, entre outros que se aplicam a este estudo.

Algumas características pessoais, que são consideradas ideais para um profissional cirurgião, como personalidade forte, autocontrole, mente questionadora, liderança e uma certa agressividade, são vistas como qualidades inerentes ao gênero masculino e ausentes nas mulheres ${ }^{4}$. A secretária-geral do Sindicato dos Médicos de São Paulo, Denize Ornelas, relata que, desde o primeiro ano da faculdade, é reforçado o discurso velado de que para serem cirurgiãs ou ortopedistas, elas (futuras médicas) precisam deixar de lado suas vidas pessoais, terem posturas masculinizadas e, ainda, submeteremse ao assédio moral e sexual ${ }^{12}$. Certas características inerentes ao cirurgião masculino convergem com as assinaladas pelas participantes ao descreverem uma cirurgiã do gênero feminino, por exemplo, a maioria das voluntárias da pesquisa descreveu uma mulher cirurgiã como sendo "forte"; entretanto, a característica agressiva ficou como a última opção de descrição, divergindo da literatura supracitada.

Muitos pontos são levantados para a não escolha da área cirúrgica, além do falso mito da falta de força, número de anos necessários para a formação, desgaste físico e emocional na residência, e do preconceito por ser mulher ${ }^{3}$. As áreas cirúrgicas da Medicina têm um problema de aceitação da mulher. Isso acontece por um problema cultural que está enraizado nas especialidades: "aqui você não pode ficar porque você é mulher"13.

Um dos dados mais importantes gerados por esta pesquisa é o de que as médicas participantes, em sua maioria, disseram que há machismo ou preconceito por parte de pacientes ou colegas de trabalho em relação às cirurgiãs, dado que conflui com os estudos apresentados, como o estudo de Pringle ${ }^{15}$ citado por Franco ${ }^{4}$, em que o fenótipo masculino inspira $25 \%$ a mais de confiança do que o feminino. Isso significa que, para qualquer cargo que pleiteie, uma mulher precisa mostrar ser, pelo menos, $25 \%$ mais capacitada do que seu concorrente masculino mais próximo, para, assim, ter as mesmas chances de sucesso.

A conclusão do presente artigo demonstrou que fatores como: horários de plantões, preconceito e dificuldade de conciliar a demanda serviço-família são limitantes na escolha de especialidades cirúrgicas pelas médicas, além de evidenciar a crescente feminização na medicina e a discrepância no crescimento do número das mulheres nesta área. Portanto, fazem-se necessários estudos como este para que questões laborais (flexibidade de horários, igualdade salarial) e comportamentais (preconceito, machismo) sejam revistas.

Participação dos autores: Paulo D: planejamento do projeto de pesquisa, aplicação do questionário, coleta e análise de dados,redação do estudo, referências. Assis MS: planejamento do projeto de pesquisa, aplicação do questionário, coleta de dados e redação do abstract e título em inglês. Kreuger MRO: orientações iniciais, planejamento, revisão e supervisão do projeto de pesquisa.

\section{REFERÊNCIAS}

1. Teixeira SA, Ferreira SL. Discurso sobre a mulher na formação médica. Rev Saúde.Com. 2011;7(2):157-70. Disponível em: http://periodicos2.uesb.br/index.php/rsc/ article/view/203.

2. Prates PR. Mulheres médicas. Rev Soc Bras Cardiol RS. 2008;15(4):1-4. Disponível em: http://sociedades.cardiol.br/ sbc-rs/revista/2008/15/pdf/mulheres_medicas.pdf.

3. Lins CC, Cruz RAR, Brandão MCM. A mulher na neurocirurgia. 2013; Arq Bras Neurocir. 2013;32(1):7-10. Disponível em: http://files.bvs.br/upload/S/0103-5355/2013/ v32n1/a3618.pdf.

4. Franco T, Santos EG. Mulheres e cirurgiãs. Rev Col Bras Cir. 2010;37(1):72-7. http://dx.doi.org/10.1590/S010069912010000100015 .
5. Ávila CR. Formação das mulheres nas escolas de medicina. Rev Bras Educ Med. 2014;38(1):142-9. https://doi. org/10.1590/S0100-55022014000100019

6. Scheffer MC, Cassenote AJF. A feminização da medicina no Brasil. Rev Bioét. 2013;21(2):268-77. http://dx.doi. org/10.1590/S1983-80422013000200010.

7. Scheffer M, et al. Demografia médica no Brasil. São Paulo: FMUSP, CFM, CREMESP; 2018. Disponível em: http://www.flip3d.com.br/web/pub/cfm/ index $10 /$ ?numero $=15 \&$ edicao $=4278$.

8. Trindade APP, Trindade DF. Desafios das primeiras médicas brasileiras. História Ciên Ensino Construindo Interfaces. 2011;4:24-37. Disponível em: https://revistas.pucsp.br/ hcensino/article/view/6435.

9. Christopher JD, Bheeshma R, Coburn N, Nam RK, Detsky AS, Satkunasivam R. Comparison of postoperative 
Paulo D, et al. Análise dos fatores que levam mulheres médicas a não optarem por especialidades cirúrgicas

outcomes among patients treated by male and female surgeons: a population based matched cohort study. BMJ. 2017;359:j4366. doi: https://doi.org/10.1136/bmj.j4366.

10. Koeche LG, Cenci I, Bortoluzzi MC, Bonamigo EL. Prevalência de erro médico entre as especialidades médicas nos processos julgados pelo Conselho Regional de Medicina do Estado de Santa Catarina. Arq Catarin Med. 2013;42(4):4553.

11. Wirtzfeld DA. The history of women in surgery. Can J Surg. 2009;52(4):317-20. Available from: https://www.ncbi.nlm. nih.gov/pmc/articles/PMC2724816/.

12. Machado N. Com licença, esse lugar é nosso. Rev DR!
2015;87(4):12-17.

13. Aranha T. Apesar das dificuldades, há coragem. Rev DR! 2013;75(1):12-9.

14. Seemann NM, Webster F, Holden HA, Moulton CA, Baxter N, Desjardins C, Cil T. Women in academic surgery: why is the playing field still not level. Am J Surg. 2016;211(2):343-9. doi: 10.1016/j.amjsurg.2015.08.036.

15. Pringle R. Sex and medicine. Cambridge: University Press; 1998

Recebido: 25/01/2019

Aceito: 04/05/2020 